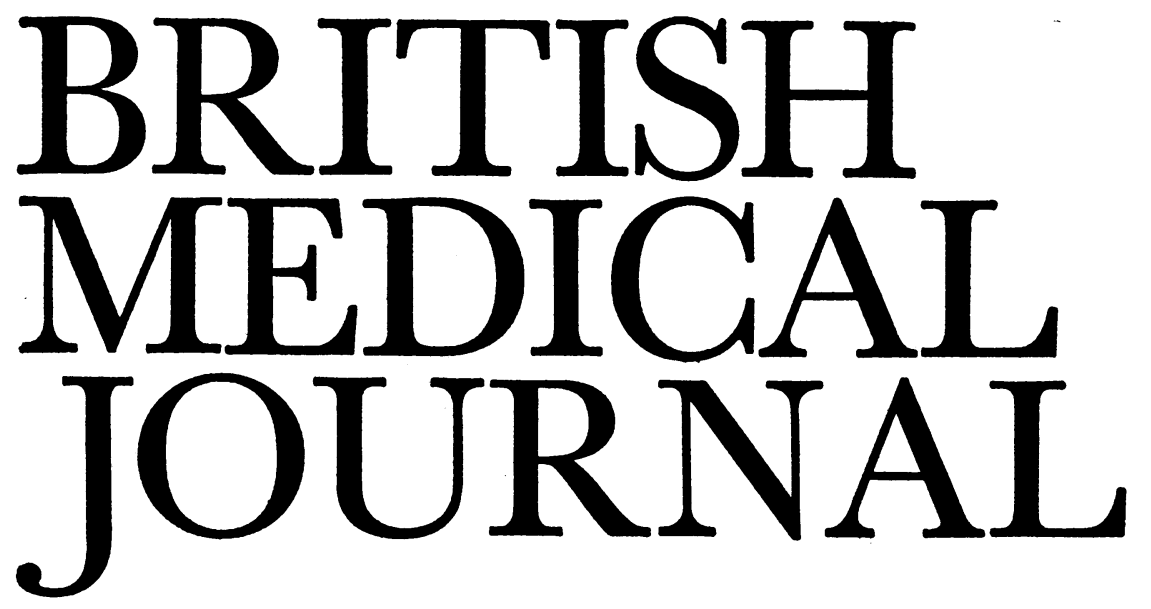

LONDON SATURDAY 18 MAY 1974

\title{
Slow Virus Infections
}

Since the discovery of Kuru in New Guinea ${ }^{1}$ and its transmission to the chimpanzee, ${ }^{2}$ research into chronic and subacute degenerative diseases of the human central nervous system has rapidly increased. Particular attention is being given to progressive multifocal leukoencephalopathy (P.M.L.) and its possible relationship to a papova-like virus; subacute sclerosing panencephalitis (S.S.P.E.) and its relationship to measles; epilepsia partialis continua and other chronic focal neurological syndromes caused by strains of the tick-borne Russian spring summer (R.S.S.) encephalitic complex; congenital rubella and cytomegalovirus in the brain $;^{3}$ subacute encephalitis with herpes simplex; encephalitis associated with adenovirus type $32 ;{ }^{4}$ presenile dementia of the spongiform CreuzfeldtJakob (C.J.) type; and Kuru. Add to these the diseases in animals such as scrapie, mink encephalopathy, and the visnamaedi complex, and it is evident that there is a wide range of hosts which may suffer potentially transmissible chronic degenerative diseases of the C.N.S. The visna-maedi complex is important because the virus can cause both chronic lung and C.N.S. disease. ${ }^{5}$ In both conditions there is cell proliferation; in the C.N.S. certain cells of the glia are affected and in the lungs lesions occur in the epithelium of the bronchioles. A recent edition of the Annals of Clinical Research ${ }^{6}$ reports on some of the important new findings in these "slow virus diseases," and it includes comment on how multiple sclerosis may have to be considered in the same context.

The group of diseases called the spongiform encephalopathies-Kuru, C. J., scrapie, and mink encephalopathy arouse particular interest. One reason for this is the extraordinary nature of the agents ${ }^{7}$ their resistance to heat inactivation, proteolytic enzymes, acetylethyleneamine, formaldehyde, RNase, DNase, and their extreme resistance to ultraviolet light inactivation. This has led to speculation that they are agents which lack nucleic acid and are able to replicate in an unusual way. Membrane disrupting substances will cause inactivation in scrapie, suggesting that a replicating membrane or a membrane associated polysaccharide may be concerned. An interesting comparison has been made recently between the scrapie agent and the very small size RNA potato-spindletuber-virus and chrysanthemum-stump-virus, sometimes termed viroids because they are much smaller than any known viruses. ${ }^{8}$ For this hypothesis to be substantiated the scrapie agent will have to be shown to possess nucleic acid. To date this seems unlikely, but nucleic acid firmly bound to membrane may still be found, and the recent finding of trace amounts of a small, single stranded DNA in scrapie brain and its apparent absence in normal brain could support this suggestion. ${ }^{6}$ Now that the C.J. agent has been transmitted to new and old world monkevs and cats ${ }^{9}$ less well financed laboratories might start trying to increase the number of isolations from this disease.

These four agents also stimulate proliferation of some glial cells, which suggests that a virus-induced oncongenic type of transformation may play a part in the pathogenesis of chronic degenerative C.N.S. diseases. For some years the pathogenesis of Kuru, scrapie, and P.M.L. has been considered in terms of excessive glial proliferation with possible transformation due to a primary viral effect, and this has been related to the pathogenesis of multiple sclerosis and the possible role which DNA and RNA viruses might play in that process. ${ }^{10} \mathrm{Glial}$ proliferation occurs both in vivo and in vitro in human and animal C.N.S. cell cultures as an apparently primary effect of infection with many differing viruses, including arboviruses of the R.S.S. complex, ${ }^{11}{ }^{13}$ West Nile, ${ }^{12}$ Semliki forest, ${ }^{11} 14$ Chikungunya, ${ }^{15}$ Quaranfil, and others such as the C.V.S. strain of rabies, ${ }^{11}$ measles, ${ }^{16}$ S.S.P.E., ${ }^{17}$ C.J. disease, ${ }^{18}$ and scrapie. ${ }^{19}$ It is not yet possible to say whether this is a primary proliferative effect of these agents on the glia or the result of a latent virus being stimulated by secondary infection to behave in an oncogenic way. Clearly some, including the papova group and adenoviruses, do have an oncogenic capacity. These two types can be recovered from cell cultures of both normal and damaged brains. ${ }^{20}$ The fact that glial proliferation and hypertrophy occurs very early, before clinical disease develops, suggests that it precedes loss of neurones. This perhaps is of the greatest significance; it emphasizes the role of the glia in diseases with secondary damage to the neurone. In special circumstances a wide range of viruses may produce gliosis either at sites of viral preference or where the maximum viral entry takes place.

These findings also support a multiple aetiology for the demyelinating diseases, as suggested by evidence that some patients with multiple sclerosis have raised antibody levels against measles, ${ }^{21}{ }^{22}$ and mumps, ${ }^{22}$ while in others different viruses have again been isolated from brain cell cultures. ${ }^{23}$ Taking into account the differing genetic susceptibilities of the hosts and the number of viruses or virus-like agents which may be concerned, it seems possible that these diseases may start by virus-induced primary gliosis, resulting in damage to the glia; this could lead to loss of myelin synthesis and its nutritional support, and eventually to loss of neurones and axons. Finally-particularly in multiple sclerosis-auto- 
immunity might develop to the resulting break-down products of myelin. ${ }^{24} \mathrm{~A}$ hypothesis of this nature has some advantages, since it brings together and gives credence to the results of many different workers in different fields. However, almost every month some new discovery is made, and our theories of today may need to be changed tomorrow to adapt to proved scientific fact.

1 Gajdusek, D. C., Transactions of the Royal Society of Tropical Medicine and Hygiene, 1963, 57, 151.

2 Gajdusek, D. C., Gibbs, C. J., jun., and Alpers, M., Nature, 1966, 209, 794.

3 Lang, D., 13th International Congress of Pediatrics, Vienna. Neurology and Psychiatry III. Vienna, Vienna Academy of Medicine, 1971.

4 Roos, R., Chou, S. M., Rogers, N. G., Basnight, M., and Gajdusek, D. C., Proceedings of the Society of Experimental Biology and Medicine, 1972, 139, 636.

5 Palsson, P. A., in Host-Virus Reactions with Special Reference to Persistent Agents, ed. G. Dick, p. 115. London published for the Royal College of Agents, ed. G. Dick, p. 115. London published for the Roy

Pathologists by the Journal of Clinical
Annals of Clinical Research, 1973, 5, 253.

7 Hunter, G. D., Kimberlin, R. H., Collis, S., and Millson, G. C., Annals of Clinical Research, 1973, 5, 262.

${ }^{8}$ Diener, T. O., Annals of Clinical Research, 1973, 5, 268.

9 Gibbs, C. J. jun., and Gajdusek, D. C., Science, 1973, 182, 67.

$10 \mathrm{Webb}, \mathrm{H}$. E., Proceedings of the Royal Society of Medicine, 1967, 60, 698

11 Z.lotnik, I., British Fournal of Experimental Pathology, 1968, 49, 555

12 Illavia, S. J., and Webb, H. E., British Medical Fournal, 1969, 1, 94

13 Illavia, S. J., and Webb, H. E., Lancet, 1970, 2, 284.

14 Zlotnik, I., Grant, D. P., and Dee Batter-Hatton, British Fournal of Experimental Pathologv, 1972, 53, 125.

15 Precious, S. W., Ph.D. thesis, University of London, 1973.

16 Webb, H. E., Illavia, S. J., and Laurence, G. D., Lancet, 1971, 2, 4.

17 Katz, M., Koprowski, H., and Moorhead, P., Experimental Cell Research, $1969,57,149$.

${ }^{18}$ Hooks, J., Gibbs, C. J. jun., Chcpra, H., Lewis, M., and Gajdusek, D. C., Science, 1972, 176, 1420 .

19 Caspary, E. A., and Bell, T. M., Nature, 1971, 229, 269.

20 Gaidusek, D. C.., Annals of Clinical Research, 1973, 5, 254.

21 Salmi, A. A., Annals of Clinical Research, 1973, 5, 319.

22 Millar, J. H. D., et al., British Medical fournal, 1971, 2, 378.

23 Ter Meulen, V., Muller, D., Kackell, Y., Katz, M., and Meyermann, R., Lancet, 1972, 2, 1172 .

24 Webb, H. E., in Multiple Sclerosis, Progress in Research, Vol. 3. ed. E. J Field, T. M. Bell, and P. R. Carnegie, p. 8. Amsterdam, North Holland Publishing Company, 1972.

\section{Kidneys from Living Donors}

Kidney transplant centres in Britain mostly use kidneys obtained from cadavers. Kidneys from living donors are used only occasionally, except in the Newcastle unit, where 51 transplanted kidneys have been obtained from volunteer blood relatives, constituting about $25 \%$ of the transplants performed.

The same trend is apparent in European countries, and recent figures published by the European Dialysis and Transplant Association show that in recent years about $90 \%$ of all transplants recorded in Europe and Britain have been from cadaveric donors. ${ }^{1}$ In Australasia recent figures show that $98 \%$ of renal transplants were cadaveric, but in contrast only $52: 6 \%$ of all grafts came from this source in the U.S.A., the rest being kidneys taken from living donors. ${ }^{2}$

Reluctance to use kidneys from living donors is understandable, but can be justified on two counts: firstly, the success rate of transplants from living donors is significantly better than in those taken from cadaveric donors; and secondly the immediate and long-term effects of removal of a kidney from an otherwise healthy person are negligible.

Grafts from unrelated living donors are probably unjustifiable, and the results are variable, but when kidneys are transplanted from related donors who are carefully assessed preoperatively both for histocompatibility and anatomical and physiological suitability there is no doubt that the results are excellent. Patients receiving transplants from sibling sources have at one year a patient survival of $90 \%$ and functional grafts in $75 \%$; at three years there is a slight fall to $82 \%$ patient survival and $73 \%$ functioning grafts. Kidneys from parent sources give a similar one year survival, but at three years patient survival is $70 \%$ and graft survival $63 \%$.

In contrast, cadaveric kidneys give a $70 \%$ patient survival in the best centres at one year and a graft survival of about $50 \%$, while at three years $60 \%$ of patients survive but only $42 \%$ of the grafts. With all groups, patient and graft survival continue to fall slowly at much the same rate after three years.

At present, therefore, the results of living donor transplants are better than cadaveric and in the present scarcity of cadaveric donors there seems a good case for using living donors.

Few reports of the effects of nephrectomy on living donors have been published, but a recent review by Farrell et al. ${ }^{3}$ has given the immediate and long-term results, both physical and psychological, in 135 cases. There is nothing special about the operative technique of donor nephrectomy, except that adequate exposure of the renal pedicle is essential to avoid damage to the renal vessels. Excision of the 11th rib usually gives sufficient exposure, but incision of the diaphragm and opening of the pleura may be necessary in some cases. In the series described mannitol and heparin were both given to the donor before dividing the pedicle, but blood transfusion was avoided because of the risk of hepatitis. There was no operative mortality, and the average time in hospital was 10 days. The most frequent postoperative complications were anaemia and retroperitoneal haematoma. Minor chest complications occurred in 30 cases, and psychoneurotic reactions in four.

Seventy-four patients were followed for periods ranging from three months to five years. Fourteen had some rise in the serum creatinine during the first three months after operation but it persisted in only six; the rest had normal renal function. One patient developed hypertension and two showed microscopic haematuria. One patient died of hepatitis three months postoperatively but had not received blood transfusion. One developed a melanoma and one breast cancer, and two had incisional herniae. None had regrets about the operation and all would accept the risks again.

These results are in agreement with those reported by Penn and colleagues, ${ }^{4}$ Leary and Deweerd, ${ }^{5}$ and with a series of 51 living donors followed up in Britain, ${ }^{6}$ but in none of these series was retroperitoneal haematoma a noteworthy complication, as it was with Farrell et al. This may be because heparin was not given to donors before division of the pedicle in the other series; and it does not seem necessary. In the British series $14 \%$ had minor chest complications and one had wound infection. In the long term all but five were completely well: two of these had nervous stress and three complained of discomfort in the scar. None had hypertension, and renal function was normal in 25 in whom it was studied. Only two regretted the donation of a kidney, one because of her husband's anxiety and one because of family friction, but there were no major psychological upsets.

The risk to the donor of giving a kidney to a relative in terminal renal failure is, therefore, slight, and in the context of the desperate situation in which a patient in terminal renal failure finds himself is fully justified, especially if the chance of receiving a compatible cadaveric kidney seems remote. It should not be undertaken, however, without full explanation of the risks and morbidity involved.

\footnotetext{
1 Garland, H. J., et al., Proceedings of the European Dialysis and Transplant Association, 1973, 10, xvii.

2 11th Report of Human Renal Transplant Registry, Chicago, Fournal of the American Medical Association, 1973, 226, 1197.

Farrell, R. M., Stubenbord, W. T., Riggio, R. R., and Muecke, E. C., Farrell, R. M., Stubenbord, W. T.,
Fournal of Urology, 1973, 110, 639.

4 Penn, I., Halgrimson, C. G., Ogden, D., and Starzl, T. E., Archives of Surgery, 1970, 101, 226.

5 Leary, F. J., and Deweerd, J. H., Fournal of Urology, 1973, 109, 947.

Fleming, L. B., Newcastle upon Tyne Renal Transplant Centre, personal communication, 1974.
} 